\title{
Organisation 4.0
}

\section{Liebe Leserinnen und Leser,}

die Organisation des Controller-Bereichs steht angesichts der ins Haus stehenden Transformation des Controllings vor neuen Herausforderungen. Zunächst führen Standardisierung und globale Zentralisierung von Controlling-Prozessen zu neuen Organisationseinheiten in Form von Shared Service Centers und Centers of Expertise, die wiederum in die Gesamtlandschaft der Finanzfunktion eingefügt werden müssen. Während es im Personalwesen mit dem Dave-UlrichModell eine etablierte Referenz für das Zusammenspiel von Shared Service, Centers of Expertise, Corporate Human Relations (HR) und Embedded HR gibt, orientiert man sich im Controlling eher am Beispiel anderer und an den Empfehlungen von Beratern. Das muss kein Fehler sein, die Komplexität der Aufgabe sollte aber auch nicht unterschätzt werden: Macht es Sinn, die konzeptionelle Weiterentwicklung von Systemen und Prozessen sowie die Schulung und Betreuung dezentraler Einheiten in eine separate Einheit zu packen? Welche Experten sitzen im Shared Service, welche im Center of Expertise? Und wie spielen Centers of Expertise dann mit dem Zentral-Controlling zusammen? Im Detail stellen sich viele neuen Fragen.

Doch damit nicht genug: Neben Shared Service und Centers of Expertise führt das Phänomen Big Data in immer mehr Unternehmen zur Gründung sogenannter Data Science Centers, in denen Mathematiker, Physiker und Informatiker strukturierte und vor allem unstrukturierte Daten(berge) analysieren. Folgt man der Harvard Business Review, gehen sie dem "sexiest job of the 21st century“ nach. Und in nicht wenigen Fällen steht ihnen ein „Chief Data Officer“ vor. Spätestens hier müssen für Controller die Alarmglocken schrillen. Haben nicht wir den spannendsten Job? Ist nicht der Controller von jeher - etwas zurückhaltender formuliert - der Hüter der Zahlen? Wer, bitte schön, ist dann in Zukunft die „single source of truth“, die Controller heute für sich reklamieren? Gibt es dann zwei Wahrheiten? Oder sind Controller dabei, ins zweite Glied zu wandern? Und ganz grundsätzlich: Wie wird das Zusammenspiel von Data Scientists und Controllern organisiert? Sind Data Scientists innerhalb des Controllings angesiedelt oder an anderer Stelle? Die gängige

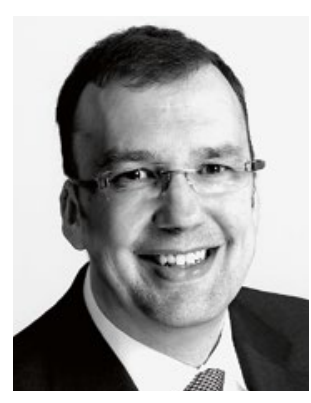

Utz Schäffer

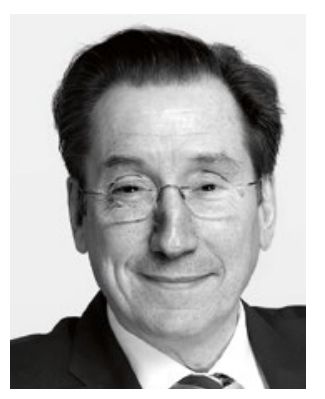

Jürgen Weber

Rhetorik strebt nach einer Zusammenarbeit auf Augenhöhe: Controller müssen demnach gar nicht alle statistischen Methoden im Detail beherrschen (tun sie ja im Regelfall auch nicht). Es reiche völlig aus, dass sie die richtigen Fragen stellen, hinreichendes Überblickswissen haben und die Sprache der Data Scientists beherrschen. Nur: Könnte die Zusammenarbeit auf Augenhöhe dann nicht auch gleich mit dem Manager erfolgen? Wo bleibt der Mehrwert der Controller?

Genau dieser Mehrwert ist es, der heute vom Management - nicht zu Unrecht - immer aggressiver eingefordert wird. Nicht zuletzt von den dezentral agierenden Business Partnern, deren organisatorische Einbindung in die Controlling Community des Hauses die nächste Herausforderung darstellt. Wie sehen die Reporting-Linien aus? Sind die Controller disziplinarisch dem Zentral-Controlling unterstellt die heute dominierende Lösung - oder diesem angesichts einer zunehmenden Dezentralisierung im Zeitalter der Digitalisierung nur über eine Dotted Line verbunden?

Sie sehen: Die Herausforderungen sind vielfältig. Wir hoffen, dass Ihnen das vorliegende Heft die eine oder andere Anregung mitgibt, und wünschen Ihnen viel Spaß bei der Lektüre.

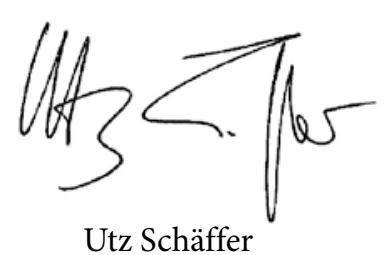

Utz Schäffer

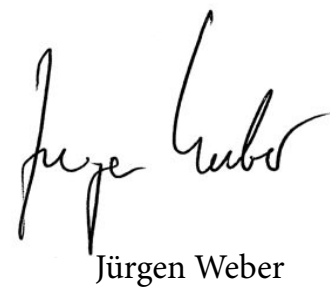

